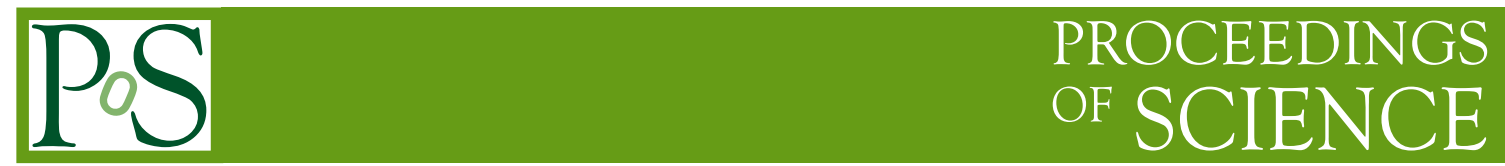

\title{
Searches with early data at CMS
}

\section{Francesco Santanastasio*University of Maryland, MD, USA}

E-mail: francesco.santanastasiodcern.ch

This paper presents an overview of prospects for searches for new physics beyond the Standard Model with early data of the CMS experiment, at the Large Hadron Collider of CERN. The results presented here are based on Monte Carlo simulations of the CMS detector, assuming 10-100 $\mathrm{pb}^{-1}$ of collected integrated luminosity and proton-proton collisions at $\sqrt{s}=7 \mathrm{TeV}$. A selection of benchmark analyses feasible with early data is discussed, including searches for new physics in the di-jet and dilepton+jets channels, the description of techniques to identify the production of heavy long-lived charged particles, and the searches for Supersymmetry in the all-hadronic and like-sign dilepton channels.

XVIII International Workshop on Deep-Inelastic Scattering and Related Subjects April 19 -23, 2010

Convitto della Calza, Firenze, Italy

\footnotetext{
* Speaker.
} 


\section{Introduction}

This paper presents a brief overview of prospects for searches for new physics, beyond the Standard Model (SM) of fundamental interactions, feasible with early data of the CMS experiment [1], at the Large Hadron Collider (LHC) of CERN. The results summarized here are based on a detailed Monte Carlo (MC) simulation of the CMS detector, assuming order of $10-100 \mathrm{pb}^{-1}$ of recorded integrated luminosity and proton-proton collisions at a center-of-mass energy of $\sqrt{s}=$ $7 \mathrm{TeV}$. The estimates of the physics reach at $7 \mathrm{TeV}$ are based on extrapolations from the existing studies at higher energies $(\sqrt{s}=10 \mathrm{TeV}$ or $14 \mathrm{TeV})$, by applying simple scaling of cross sections for signal and backgrounds as described at [2]. A selection of several benchmark analyses with different experimental signatures is discussed in the following sections.

\section{Di-jet channel}

Several theoretical models predict the existence of new high mass resonances decaying to two jets. Even if the energy of the LHC is not sufficient to directly produce these new particles, the new physics might still appear as a quark contact interaction, and the LHC experiments should be able to identify its signature by looking at di-jet events. We discuss here the CMS analysis [3] of the di-jet ratio ${ }^{1}$ used to identify the presence of contact interactions. The most sensitive search for contact interactions at the Tevatron gives an exclusion at 95\% C.L. on the contact interaction scale of $\Lambda^{+}<2.4 \mathrm{TeV}$ [4]. Figure 1 (Left), illustrates the sensitivity of this measurement to contact interactions for different values of $\Lambda^{+}$for a scenario at $\sqrt{s}=14 \mathrm{TeV}$. The sensitivity to new physics in this channel at $7 \mathrm{TeV}$ is also expected to be rather high and is currently being estimated.

\section{Heavy long-lived charged particles and stopped R-Hadrons}

Some models of new physics predict the existence of exotic particles that are heavy (mass of hundreds of $\mathrm{GeV} / \mathrm{c}^{2}$ ), long-lived (enough to decay outside of the detector) and charged [5]. Heavy long-lived particles with hadronic nature, such as gluinos or stops, hadronize in flight, forming meta-stable bound states with quarks and gluons (so called R-Hadrons).

Such particles can be distinguished from SM particles by exploiting their unique signature: a low velocity $(\beta=p / E<1)$ associated with a high momentum (few hundreds of GeV/c). Two offline methods are used to measure $\beta$ [6]: $\beta_{\mathrm{DT}}$ is derived from the time delay of the arrival of the particle at the muon chambers, while $\beta_{\mathrm{Tk}}$ is obtained from the $d E / d x$ measured in the silicon tracker. The $d E / d x$ measurement have been commissioned with low energy hadrons using early collision data, as described at [7]. The analysis results show that gluino and stop with mass of about $500 \mathrm{GeV} / \mathrm{c}^{2}$, and $350 \mathrm{GeV} / \mathrm{c}^{2}$, respectively, can be excluded at $95 \%$ C.L. with $100 \mathrm{pb}^{-1}$ of data at $7 \mathrm{TeV}$ (see Figure 1, Right).

R-Hadrons lose energy via electromagnetic and nuclear interactions while traveling inside the CMS detector. For low- $\beta$ R-hadrons, this energy loss is sufficient to bring a significant fraction of the produced particles to rest inside the CMS detector volume (in particular in the hadronic

\footnotetext{
${ }^{1}$ The di-jet ratio is an effective angular variable defined as $N(|\eta|<0.7) / N(0.7<|\eta|<1.3)$, where $N$ is the number of di-jet events with both jets satisfying the pseudo-rapidity requirements in parentheses.
} 
calorimeter HCAL). These "stopped" R-hadrons will decay seconds, day, or weeks later (accordingly to the "unknown" lifetime). These decays will be out-of-time with respect to the LHC collisions and may well occur at times when there are no collisions (e.g., beam gaps) or when there is no beam in the LHC machine (e.g., inter-fill period).

For the online selection of these events, the CMS experiment developed a dedicated calorimeter trigger. The observation of such decays in a form of an isolated jet in HCAL, in what should be a quiet detector (save for the occasional cosmic ray or noise from the calorimeters), would be an unambiguous discovery of new physics [8]. Figure 2 (Left) shows that few weeks of data taking at $7 \mathrm{TeV}$, with an instantaneous luminosity of $10^{32} \mathrm{~cm}^{-2} \mathrm{~s}^{-1}$, will be sufficient to discover a long-lived gluino of $300 \mathrm{GeV} / \mathrm{c}^{2}$ over a large range of lifetimes.
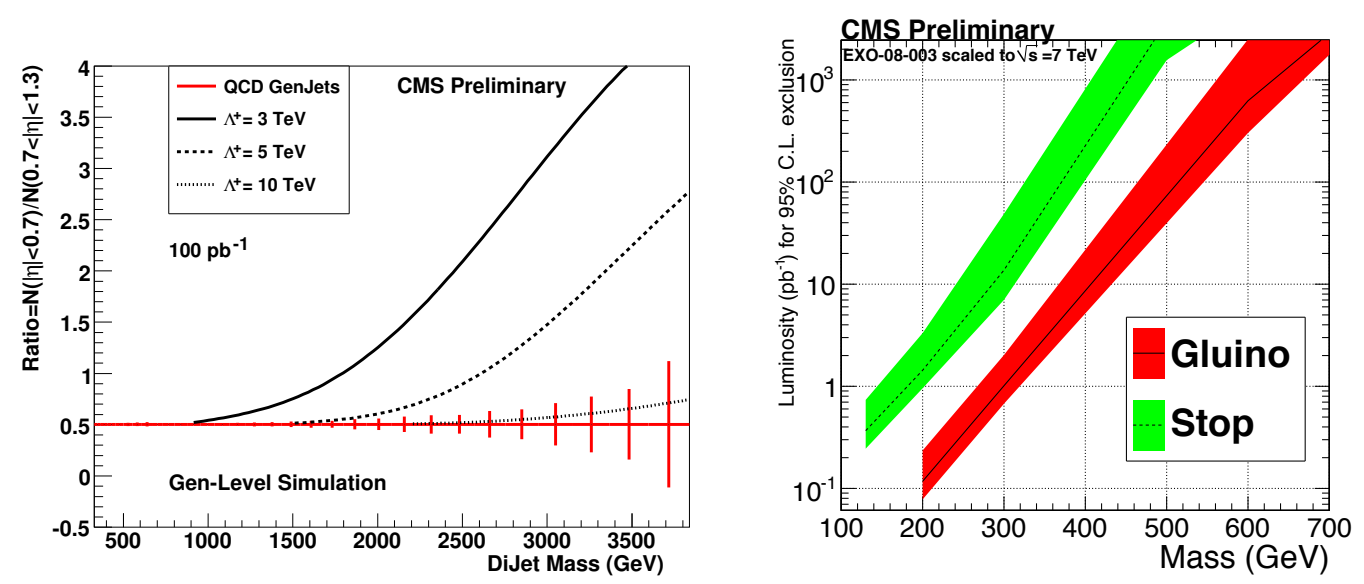

Figure 1: Left: Di-jet ratio as a function of di-jet mass in presence of contact interactions at different energy scales $\Lambda^{+}$, and for QCD multi-jet background at $\sqrt{s}=14 \mathrm{TeV}$. Right: 95\% C.L. limit for HSCP searches at $7 \mathrm{TeV}$. Tracker-only analysis (i.e. $\beta_{\mathrm{Tk}}$ measurement) is used. Current lower limit of stop mass is around $250 \mathrm{GeV}$ [9].

\section{Dilepton+jets channel}

The experimentally observed symmetry between families of leptons and quarks in the SM has motivated the search for leptoquarks $(L Q)$, hypothetical bosons carrying both quark and lepton quantum numbers that decay in a lepton and a quark. The pair production of the first (second) generation scalar $L Q$ has been studied in CMS [10], in the final state with 2 high $p_{T}$ electrons (muons) and 2 high $p_{T}$ jets. Figure 2 (Right) shows the CMS exclusion reach for the first generation $L Q$ analysis with $100 \mathrm{pb}^{-1}$ at $\sqrt{s}=7 \mathrm{TeV}$ and the current Tevatron limit. Similar reach is obtained in the $\mu \mu j j$ channel.

\section{Supersymmetry}

CMS will perform a broad range of searches for supersymmetric (SUSY) particles. The initial searches will be performed in a variety of inclusive final states involving jets, leptons, photons and missing transverse energy. Background will be determined using data-driven methods whenever possible, with multiple methods for crosschecks. 

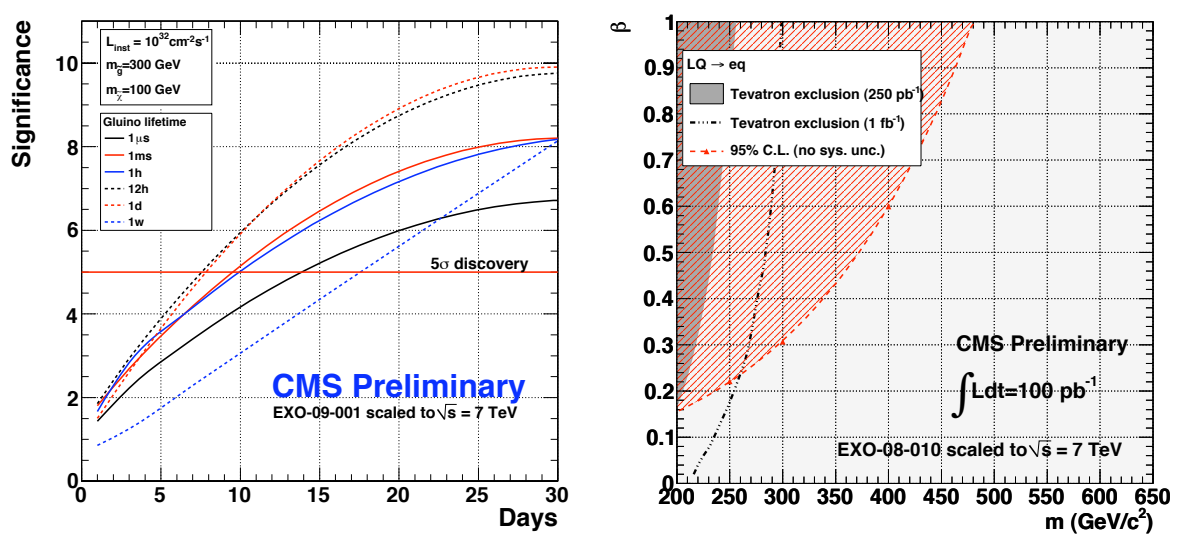

Figure 2: Left: The discovery potential for a long-lived $300 \mathrm{GeV} / \mathrm{c}^{2}$ heavy gluino stopping in the CMS calorimeter in a $7 \mathrm{TeV}$ run at an instantaneous luminosity of $10^{32} \mathrm{~cm}^{-2} \mathrm{~s}^{-1}$ as a function of the data-taking duration. Right: The 95\% C.L.limit for first generation leptoquarks in the ee jj channel as a function of their branching fraction into electron and quark for $100 \mathrm{pb}^{-1}$ of data at $7 \mathrm{TeV}$.

Theorists have noted that the models commonly adopted for use as benchmarks, such as mSUGRA, do not span the full range of reasonable phenomenological patterns for which experiments should search. As a consequence, it is important to design searches that are as generic as possible. Nevertheless, the sensitivity of the analyses for $100 \mathrm{pb}^{-1}$ and $1 \mathrm{fb}^{-1}$ at $7 \mathrm{TeV}$ [2] is presented in Figure 3, for both all-hadronic and like-sign dilepton signatures, using a scan over mSUGRA parameters, since it allows direct comparison with existing results from Tevatron and LEP experiments.
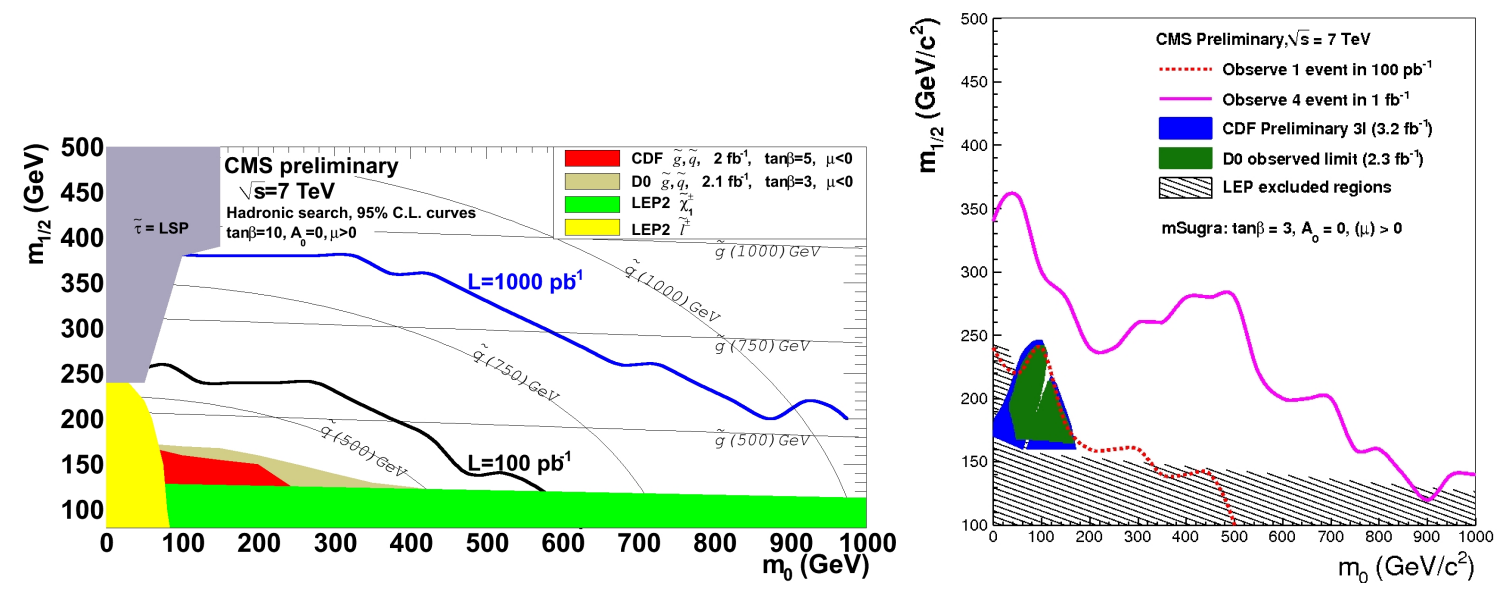

Figure 3: Left: Estimated 95\% C.L. exclusion limits for the all-hadronic SUSY search, expressed in mSUGRA parameter space. Right: Estimated 95\% C.L. exclusion limits for the like-sign dilepton SUSY search, expressed in mSUGRA parameter space. The expected standard model background at $100 \mathrm{pb}^{-1}$ $\left(1 \mathrm{fb}^{-1}\right)$ is 0.4 (4.0) events; an observed yield of 1 event (4 events) is assumed for the purpose of setting these exclusion limits. 


\section{Conclusions}

At the time of the conference (April 2010), the LHC delivered a fraction of $\mathrm{nb}^{-1}$ of pp collisions at $\sqrt{s}=7 \mathrm{TeV}$. This amount of integrated luminosity is not sufficient to improve the existing constrains on new physics set by other experiments; anyway, for some of the analyses presented here, this "turning point" could already be reached with $1-10 \mathrm{pb}^{-1}$ of data.

The LHC is expected to deliver approximately $100 \mathrm{pb}^{-1}$ of integrated luminosity between the end of 2010 and the beginning of 2011 , and $1 \mathrm{fb}^{-1}$ by the end of 2011 . This large $7 \mathrm{TeV}$ dataset will allow to set stringent limits on many exotic theoretical models, and, if Nature is kind to us, to even observe the first evidence of new physics beyond the SM.

\section{References}

[1] R. Adolphi et al. [CMS Collaboration], "The CMS experiment at the CERN LHC," JINST 3, S08004 (2008).

[2] CMS Collaboration, “The CMS physics reach for searches at 7 TeV,” CMS-NOTE-2010-008 (2010).

[3] CMS Collaboration, "CMS Search Plans and Sensitivity to New Physics using Dijets," CMS-PAS-SBM-07-001 (2007), and references on the theory therein.

[4] B. Abbott et al. [D0 Collaboration], Phys. Rev. Lett. 82, 2457 (1999) [arXiv:hep-ex/9807014].

[5] M. Fairbairn, A. C. Kraan, D. A. Milstead, T. Sjostrand, P. Z. Skands and T. Sloan, "Stable massive particles at colliders," Phys. Rept. 438, 1 (2007) [arXiv:hep-ph/0611040].

[6] CMS Collaboration, "Search for Heavy Stable Charged Particles with 100 inverse picobarns and 1 inverse femtobarn in the CMS experiment," CMS-PAS-EXO-08-003 (2009)

[7] CMS Collaboration, "Tracking and Vertexing Results from First Collisions," CMS-PAS-TRK-10-001 (2009).

[8] CMS Collaboration, "Searching for Stopped Gluinos during Beam-off Periods at CMS," CMS-PAS-EXO-09-001 (2009)

[9] V. M. Abazov et al. [D0 Collaboration], "Search for Long-Lived Charged Massive Particles with the D0 Detector," Phys. Rev. Lett. 102, 161802 (2009) [arXiv:0809.4472 [hep-ex]]. T. Aaltonen et al. [CDF Collaboration], "Search for Long-Lived Massive Charged Particles in $1.96 \mathrm{TeV} \bar{p} p$ Collisions," Phys. Rev. Lett. 103, 021802 (2009) [arXiv:0902.1266 [hep-ex]].

[10] CMS Collaboration, "Search for Pair Production of First Generation Scalar Leptoquarks at the CMS Experiment," CMS-PAS-EXO-08-010 (2009) CMS Collaboration, "Search for Second Generation Scalar Leptoquarks with the CMS Detector," CMS-PAS-EXO-09-010 (2009), and references on the model therein. 